\title{
Innovative Application of Glass Materials in Modern Decoration Modeling
}

\begin{abstract}
Yunxiu $\mathrm{Wu}^{1, *}$
${ }^{1}$ Wuhan Textile University, Wuhan, Hubei 430073, China

"Corresponding author. Email: 670138792@qq.com

ABSTRACT

With the development of science and technology, new materials emerge in endlessly, so that various decorations of different materials appear in modern life, and decorations of different materials play different decorative effects in modern life. Decorations made of glass materials are no exception. Decorations made of this material give people a sense of transparency, ease and pleasure, and they have different decorative effects in different environments. Starting from the different shapes of glass materials, this article mainly discusses point-shaped glass materials, linear glass materials, planar glass materials and glass materials of different colors, highlighting that the decoration of glass materials and different shapes and colors has the characteristics that other materials do not have, and can play different decorative effects in different environments in modern life.
\end{abstract}

Keywords: glass decoration, point-shaped glass decoration, linear glass decoration, planar glass decoration, innovative application

\section{INTRODUCTION}

With the development of science and technology, the continuous emergence of new materials makes the decoration materials in modern life innovative. Whether it is a point-shaped material, a linear material, a bulk material, or a planar material, whether it is the texture on the surface, or the organizational structure inside the material, etc., it has become more beautiful and more sophisticated with the emergence of high technology. Also, the shape is more and more novel and unique. And glass materials are no exception. With the emergence of modern high technology, there are various kinds of glass materials, such as transparent types, various patterns, various colors and so on. With the social progress, people's material life has been guaranteed, and people's quality of life has been improved to varying degrees. People began to pursue spiritual life while the aesthetic concept has also been improved, and the requirements for the shape, material and color of various decorations have also increased. The unique texture and aesthetic feeling of glass materials will become the standard of high life, high taste and high quality that modern people pursue. The innovative application of glass materials in modern decoration not only increases the decoration materials of modern design, breaks many limitations of the original materials, but also adds the natural aesthetic feeling of the shape and color of modern glass materials, which can meet people's life and aesthetic needs.
High technology and social progress have brought fierce competition to the modern design industry. All walks of life can quickly occupy the market for the development of their own industry. Everyone is constantly trying to improve the quality, texture, form, beauty and so on of their products. The design industry is no exception. Designers are constantly discovering new materials in order to find inspiration. They try their best to design unique and novel products to meet the needs of modern people, and the choice of materials has become their primary task. At the same time, they design novel and unique products with new materials to meet the needs of consumers. The quality of the material will directly affect the modeling effect of the decoration, and will also directly affect the success of the design. The unique and exquisite texture of the glass material can't be possessed by other materials. Applying the natural texture of the glass material to the decorations in modern life will add natural and simple texture to the modern decorations. The biggest feature of glass materials is that they are transparent and will melt into a flowing liquid at a certain high temperature. This liquid can be made into various shapes, and become different shapes of solids after modeling cooling. Moreover, add the required colors under high temperature, the glass material can also be made into decorations of various colors, which is also not available for other materials. People can use these unique, novel, natural and simple characteristics, and apply it in modern life decorations. And then, 
unexpected decorative effects and beauty can be obtained.

\section{OVERVIEW OF GLASS MATERIALS}

Glass materials can be divided into tempered glass, frosted glass, sand-blasted glass, patterned glass, laminated glass, bullet-proof glass, hot bending glass, hollow glass, glass brick and cellophane according to their properties. Also, they can be divided into pointshaped glass, linear glass and planar glass according to their forms. The glass in this article can be used for all kinds of decorations. Now, from the perspective of the shape of glass, people can apply point-shaped, linear and planar glass materials to the modeling design of modern decorations, which can increase the aesthetic feeling of modern decorations, expand the design thinking of designers, expand the range of design subjects, and increase the source of materials for modern design. The point-shaped glass, linear glass and planar glass are the same as the design principles of point, line and plane. They have beautiful form rules. They are indispensable elements in modern design and cannot be replaced by other elements. Point-shaped glass material, linear glass material and planar glass material all have their own characteristics. When they are used in the modeling of modern decorations, different effects will be obtained, adding the unique beauty of natural texture of decorations. How to apply point-shaped glass material, linear glass material and planar glass material in modern decoration design is an important problem for modern designers to solve.

\section{APPLICATION OF POINT-SHAPED GLASS MATERIAL IN MODERN DECORATION}

The point-shaped glass material has the sense of aggregation, independence and small and exquisite. The glass decorations can give people a feeling of delicacy, compactness and embellishment. Putting this kind of decorations in different environments has different decorative effects. How to apply point-shaped glass material in modern decoration? Let's talk about the process from designing to making decorations. It is necessary to design the modeling of the decoration, draw the design sketch, put the glass material in high temperature, wait for it melting into fluid, and then follow the modeling of the decoration, and slowly use point to shape until it is shaped. The shape of the decoration is consistent with that of the painting. After cooling, it becomes a solid point-shaped glass decoration. Different from other materials, point-shaped glass decorations have a sense of transparency, purity, cleanness and lightness. When they are placed in strong light, they give out dazzling light. While the decorations of other materials are thick, strong and stable, this kind of decorations cannot reflect dazzling light like point-shaped glass decorations. Putting it in different environment will make a sense of being small and exquisite, as shown in the "Fig. 1":

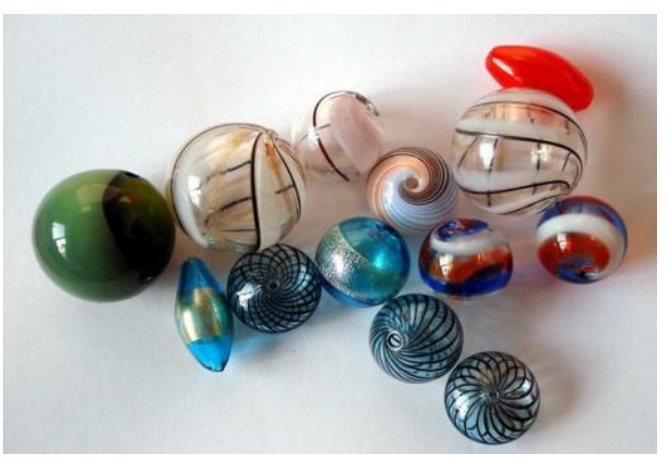

Fig. 1. Point-shaped glass decorations.

\section{APPLICATION OF LINEAR GLASS MATERIAL IN MODERN DECORATION}

Linear glass material has the sense of speed, lightness and fineness. After applying this material in decoration, it has different decoration effects in different environments. How are linear glass decorations designed and made? Now let's take a look at the specific design and manufacturing operation. According to the needs of decoration in different environments, it is required to design the modeling of the decoration, draw the effect picture of the decoration, and melt the glass material at a certain high temperature. According to the designed effect picture of the decoration, it is better to make the linear glass decoration step by step along the effect picture. This kind of linear glass decoration is different from that of other materials. The linear glass decoration has the sense of being thin, transparent and speed. It gives people a relaxed, fine and pleasant feeling when it is placed in different environments. And it will give out dazzling light when it is placed in strong light. Linear decorations of other materials are thick and strong, having no sense of transparency. Under strong light, they will not give out beautiful light. The linear glass decorations are as follows: ("Fig. 2")

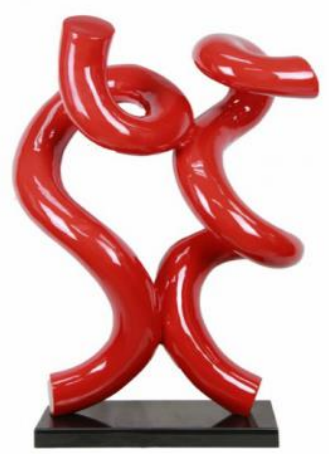

Fig. 2. Linear glass decorations 


\section{APPLICATION OF PLANAR GLASS MATERIAL IN MODERN DECORATION}

Planar glass decorations are thick, stable and strong, and have different decorative effects in different decorative environments. How shall people design and make a planar glass decoration? It is necessary to see the design and manufacturing operation. According to the environment, it is required to design the decorations, draw effect picture of the decoration, melt the glass materials at a certain high temperature, and follow the design sketch of this decoration to make a planar glass decoration. After the planar glass decoration cools and solidifies, it is completed. The products are placed in different environments for decoration, giving people a sense of stability, calmness, maturity and down-to-earth, and they will emit beautiful light under the shiny light. This planar glass decoration is different from that of other materials. The planar decoration of other materials does not emit any light under the light, and they are bulky, strong and dull. The planar glass materials are as the following: ("Fig. 3")

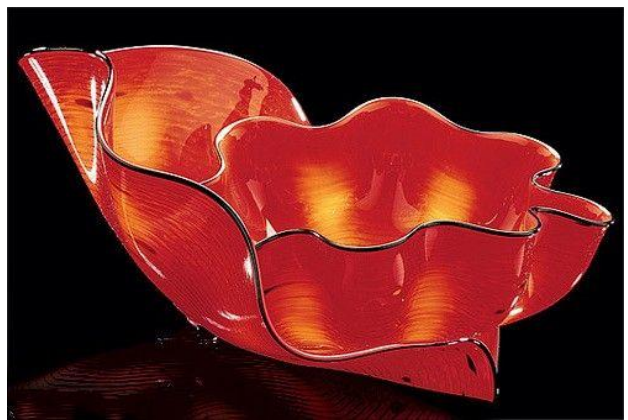

Fig. 3. Planar glass decoration

\section{APPLICATION OF MULTI-COLOR GLASS MATERIALS IN MODERN DECORATIONS}

The glass material can also be transformed into colorful decorations. This multi-colored glass decoration has colorful beauty. When these multiple colors are combined, they will emit a variety of different colors of transparent light lines under strong light, making beautiful light source. How is this colorful glass decoration designed and made? Let's take a look at the specific design and production process. First of all, it is necessary to design the decoration according to the needs of the decorative environment, and draw the design sketch, melt the glass material under a certain high temperature, add various different colors to the melted liquid, and then make the products according to the effect picture drawn first. After cooling, colorful glass decorations are produced. This colorful glass decoration will make people enter a rich and colorful world, and emit a colorful light under strong light. The decoration of other colorful materials is not as harmonious as that of colorful glass materials, and the connection between colors is not natural. The colorful glass material not only adds special decorative effects to the decorative environment, but also increases the beauty of the rich colors of the decorations.

When designing colorful glass decorations, people can add various required colors during the production for decoration. If people want to design a comprehensive colorful glass decoration such as red, blue, yellow, white, etc., it is necessary to add red, blue, yellow, and white when melting glass materials at a certain high temperature, and then make the production according to the effect picture of the decoration. After cooling, the required red, blue, yellow, white and other comprehensive colorful glass decorations are made. A variety of red, blue, yellow, white and other colorful glass decorations are particularly beautiful in a certain decorative environment, as shown in the following figure: ("Fig. 4")

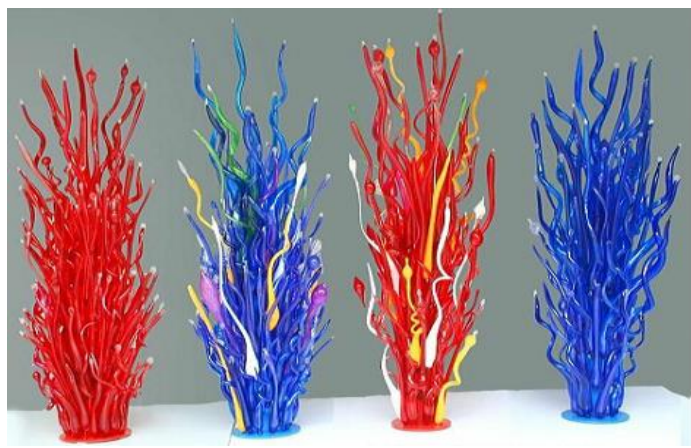

Fig. 4. Multi-color glass decorations.

\section{CONCLUSION}

From the above, it can be concluded that whether it is a point-shaped glass decoration, a linear glass decoration, or a planar glass decoration, it is very different from other material decorations. Glass material decorations have their own unique characteristics of transparency, lightness, brightness and smoothness, and can shine under strong light. The uniqueness is unmatched by other materials, and the decoration of each material has its own characteristics. Designers can make the design according to the different characteristics of the materials and the decoration needs of different environments. Some environments need glass material decorations, and some need stone decorations, wood decorations, bamboo decorations, etc.

With the advent of high technology, people's lives have undergone earth-shaking changes. People began to pursue spiritual life under the conditions of material life satisfaction, and people's aesthetics have also been 
improved in the process of pursuing spiritual life. With the exchanges between China and foreign countries, foreign products continue to flow into China. People are particularly picky about Chinese products. In this environment, China has to adopt new technologies to create better and more beautiful new materials, and produce more beautiful and better decorations to meet the needs of modern people's spiritual life.

\section{References}

[1] Wu Ouhong. "Glass Decoration Design", Tsinghua University Press, Beijing, April 2014. (in Chinese)

[2] Wang Chengyu. "Art Glass and Decorative Glass", Chemical Industry Publishing, 2009. 4. (in Chinese)

[3] Baidu Library. "Glass Technology", 2018. 7. (in Chinese)

[4] Shen Congwen. "Historical Discussion of Glass Craft", Fine Arts Research, 2016. 12. (in Chinese)

[5] "Application of Glass Materials in Environmental Art Design" Liu Haitao, Baidu HowNet, 2015.10. (in Chinese)

[6] Ren Yu. "Discussion on the Application of Decorative Glass Materials in Modern Interior Design", "Art Technology", 2018.4. (in Chinese) 